\title{
Anisotropy in the dielectric spectrum of hydration water and its relation to water dynamics
}

\author{
Stephan Gekle ${ }^{1}$ and Roland R. Netz ${ }^{2}$ \\ ${ }^{1}$ Physik Department, Technische Universität München, 85478 Garching, Germany \\ ${ }^{2}$ Fachbereich Physik, Freie Universität Berlin, 14195 Berlin, Germany
}

(Received 3 July 2012; accepted 17 August 2012; published online 13 September 2012)

\begin{abstract}
Proteins, molecules, and macromolecular assemblies in water are surrounded by a nanometer-sized hydration layer with properties very different from bulk water. Here, we use classical molecular dynamics simulations to study the dielectric response of hydration water next to hydrophobic and hydrophilic planar surfaces. We find the interfacial dielectric absorption of water to be strongly anisotropic: compared to bulk water, which shows a broad dielectric absorption maximum around $15 \mathrm{GHz}$ in the imaginary part of the dielectric function, the absorption for electric fields parallel to the surface is of similar strength and shows a slight redshift, while for perpendicular electric fields it is strongly attenuated and blueshifted. This anisotropy is generic for hydrophobic and hydrophilic surfaces. From our spatially resolved dielectric functions and a modified Maxwell-Garnett theory that accounts for anisotropic hydration layers around spherical particles, the dielectric absorption of solutions of organic molecules and micelles is derived to exhibit the experimentally known attenuation in combination with a redshift. These two features are traced back to the subtle interplay of interfacial depolarization effects and the dielectric anisotropy in the hydration layer. By a detailed analysis of the individual water molecule dynamics the perpendicular blueshift is shown not to be linked to accelerated water reorientation, but rather to dielectric boundary effects. Carefully conducted angularly resolved experiments at planar aqueous interfaces will be able to resolve this dielectric anisotropy and thus to confirm the subtle connection between spectral absorption features and the molecular water dynamics in hydration layers. () 2012 American Institute of Physics. [http://dx.doi.org/10.1063/1.4749380]
\end{abstract}

\section{INTRODUCTION}

The frequency-dependent dielectric function, or, equivalently, the electromagnetic absorption spectrum, gives a welldefined finger print of material properties over a wide range of time scales. Spectral features in the $\mathrm{GHz}$ and $\mathrm{THz}$ range draw a detailed picture of the relaxational dynamics of the water hydrogen-bond network and allow to gain insight into the hydration layer properties around proteins, membranes, molecules, and ions. ${ }^{1-5}$ Additional insight from polarizationresolved pump-probe experiments, nuclear magnetic resonance (NMR) and Raman studies has led to the notion that hydration water dynamics is finely coupled to the solute properties. ${ }^{6-9}$ This progress was made possible by simulations that nowadays allow to derive absorption spectra in the $\mathrm{GHz}$ as well as THz to infrared regimes by a combination of classical and ab initio techniques. ${ }^{10-13}$ Dielectric relaxation and $\mathrm{THz}$ spectroscopy experiments ${ }^{14,15}$ as well as molecular dynamics (MD) simulations ${ }^{16}$ have consistently found a shift of the dielectric absorption peak from the well-known $15 \mathrm{GHz}$ in bulk water to lower frequencies in the solvation shell around small organic molecules. In addition, the strength of the dielectric response was found to be diminished at all frequencies. This observation is commonly interpreted as a slowing down of the collective dynamics of hydration water and relevant in light of the role of water for protein aggregation, recognition, and folding. ${ }^{17-20}$ But one notes that the interpretation of experiments in terms of microscopic detailed pic- tures is not always straightforward and, therefore, a number of open questions about the comparison of different experimental techniques remain. ${ }^{21}$

In this work, we use classical molecular dynamics simulations to show that the collective water dynamics in the solvation shell possesses a richer structure than previously thought. We find that the dielectric function for planar hydrophobic and hydrophilic interfaces is highly anisotropic: the response to electric fields applied parallel to the surface is very different from the perpendicular one. The parallel imaginary component shows a slight redshift, i.e., an absorption peak shifted to lower frequencies, and only a small change of the absorption strength. For the perpendicular imaginary component, the response is considerably diminished and shows a pronounced blueshift. These spectral features are only indirectly related to the microscopic water dynamics, in contrast to what one might naively assume: We find only mild changes of the reorientation dynamics of interfacial water molecules. Rather, the stark contrast between parallel and perpendicular spectra is caused by dielectric boundary effects, which illustrates the subtle relation between spectroscopy and the molecular dynamics. We note that the anisotropy we are dealing with in this paper is induced by the presence of an interface and thus is distinct from the symmetry breaking between the longitudinal and the transversal components of the wave vector dependent dielectric function that is present also in bulk. ${ }^{22-24}$ 
The analysis of experimental $\mathrm{GHz}$ and $\mathrm{THz}$ absorption spectra in terms of hydration layer properties is complicated due to the superimposed spectral contributions of solute and water and in particular due to the presence of interfacial depolarization effects, which can lead to erroneous interpretations in terms of spurious interfacial effects. We combine our anisotropic interfacial dielectric profiles extracted from molecular dynamics simulations with a modified MaxwellGarnett theory (MGT) for the effective absorption of a solution of particles that accounts for anisotropic hydration layers around spherical particles. Our results exhibit an overall decrease of the absorption and a redshift of the absorption maximum, in agreement with the general trend in experiments, ${ }^{14,15}$ which shows that both parallel and perpendicular components of the dielectric function are essential in order to reproduce experimental findings. In particular, we nearly quantitatively account for experimental $\mathrm{GHz}$ spectroscopy results for micellar solutions. ${ }^{25}$ Experimentally, resolving the anisotropic absorption spectrum and, therefore, a direct verification of our results will be possible by anisotropic dielectric relaxation spectroscopy on oriented lipid bilayer stacks ${ }^{4,26}$ or surface-sensitive measurement techniques such as time-resolved pump-probe second harmonic ${ }^{27}$ or sum frequency generation on single planar interfaces. ${ }^{28}$ Alternatively, one could use oriented probe molecules in order to study the anisotropic absorption at interfaces. ${ }^{29}$

\section{METHODS}

The setup of our molecular dynamics simulations is illustrated in Fig. 1(a). A water slab with varying thickness $d$ is confined at fixed pressure $P=1 \mathrm{~atm}$ and temperature $T=300 \mathrm{~K}$ between two diamond surfaces. To model a hydrophobic surface, the uncharged surface carbon atoms are terminated by hydrogen atoms, for a hydrophilic surface $25 \%$ of the terminating hydrogens are replaced by hydroxyl groups, ${ }^{30}$ as shown in Figs. 1(b) and 1(c), respectively. The contact angle on the hydrophobic diamond is $101^{\circ}$, while the hydrophilic substrate exhibits complete wetting. ${ }^{31}$
We use the SPC/E water model ${ }^{32}$ together with the GROMOS force field for the carbon atoms. The dielectric spectrum of SPC/E water in bulk exhibits good agreement with experimental data up to $100 \mathrm{GHz}$ as shown in Appendix A. Similarly, good bulk spectra have been obtained from the TIP4P potential. ${ }^{33}$ Simulations are run with the GROMACS molecular dynamics package. ${ }^{34}$ Some simulations are verified using LAMMPS. ${ }^{35}$ The hydrogen atoms terminating the hydrophobic diamond have no partial charges and no Lennard-Jones interaction. For the hydroxyl groups on hydrophilic surfaces, we use the same Lennard-Jones parameters and partial charges as in previous work. ${ }^{30}$ We first run equilibration simulations in which the diamond structure is relaxed and the vertical slab size is adjusted to atmospheric pressure. In production runs, the diamond (including the terminating $\mathrm{H}$ and $\mathrm{OH}$ ) is then kept frozen which leads to a random, but non-zero static substrate polarization due to oriented $\mathrm{OH}$ groups. This polarization is subtracted in Eq. (2) (see below) and thereby does not influence the resulting spectra. The number of water molecules ranges from 289 in the thinnest up to 3956 in the thickest slab. The lateral system size is $3.2 \times 3.2 \mathrm{~nm}^{2}$ for the hydrophobic and $4.2 \times 4.2 \mathrm{~nm}^{2}$ for the hydrophilic slabs.

The temperature is kept at $300 \mathrm{~K}$ using a Berendsen thermostat, ${ }^{36}$ electrostatic interactions are calculated using particle-mesh-Ewald summation with a slab correction. ${ }^{37}$ For Lennard-Jones interactions a switched cut-off between $0.9 \mathrm{~nm}$ and $1.0 \mathrm{~nm}$ is used. In all simulations, the first $500 \mathrm{ps}$ are discarded for equilibration, total simulation durations range up to $3.5 \mu \mathrm{s}$. Time correlation functions are cut when they drop below zero (due to noise). Laplace transformations are then calculated numerically. It is verified explicitly that all dielectric spectra satisfy the Kramers-Kronig-relations. ${ }^{38}$

\section{RESULTS AND DISCUSSION}

\section{A. Anisotropic interfacial dielectric spectra}

The dielectric response of any physical system is expressed by the complex, frequency-dependent dielectric function $\epsilon(f)=\epsilon^{\prime}(f)-i \epsilon^{\prime \prime}(f)$. Using linear response
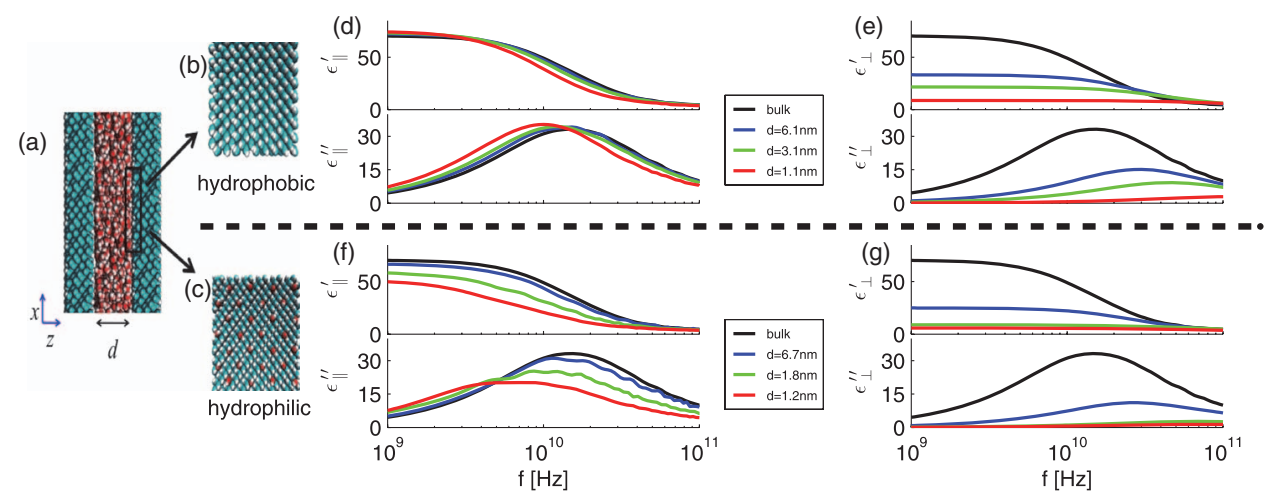

FIG. 1. (a) Our simulation setup consists of two diamond blocks which confine a water slab of thickness $d$ varying between 1.1 and 6.7 nm. The surfaces are either terminated by hydrogen atoms making them hydrophobic (b), or by hydroxyl groups making them hydrophilic (c). (d) The real and imaginary part of the frequency-dependent dielectric function parallel to a hydrophobic surface compared to that of bulk water. While the overall magnitude of the absorption peak in the imaginary part hardly changes even for the nanometer thin slab, its position is clearly shifted to lower frequencies. (e) The dielectric function for the perpendicular component looks drastically different: the response is severely attenuated for the thin slabs and the absorption peak is strongly shifted towards higher frequencies. ((f) and (g)) For the hydrophilic surface the behavior is similar (except for a slight decrease of the low-frequency parallel real response), demonstrating the generality of the observed anisotropy in the dielectric function of interfacial water. 
theory, ${ }^{33,38}$ it follows directly from the polarization timecorrelation function

$$
\Phi_{\alpha}(t)=\left\langle\boldsymbol{M}_{\alpha}(0) \cdot \boldsymbol{M}_{\alpha}(t)\right\rangle-\left\langle\boldsymbol{M}_{\alpha}\right\rangle \cdot\left\langle\boldsymbol{M}_{\alpha}\right\rangle, \quad \alpha=\|, \perp,
$$

where the angular brackets indicate an average over phase space trajectories and the term $\left\langle\boldsymbol{M}_{\alpha}\right\rangle$ accounts for a non-zero average polarization. We have split the polarization fluctuations into components parallel $\boldsymbol{M}_{\|}$and perpendicular to the planar interface $M_{\perp}$, as suggested by static results $(f=0)$ for interfacial water ${ }^{39-41}$ and frequency-dependent investigations of dipolar model fluids. ${ }^{42}$ The parallel and perpendicular dielectric functions follow as (see Appendix B for a detailed derivation)

$$
\begin{gathered}
\epsilon_{\|}(f)=1+L_{i f}\left(-\dot{\Phi}_{\|}\right) /\left(2 \epsilon_{0} k_{\mathrm{B}} T V\right), \\
\frac{\epsilon_{\perp}(f)-1}{\epsilon_{\perp}(f)}=\frac{L_{i f}\left(-\dot{\Phi}_{\perp}\right)}{\epsilon_{0} k_{\mathrm{B}} T V} .
\end{gathered}
$$

Here, $L_{i f}$ denotes the Laplace transformation given by $L_{i f}(g(t))=\int_{0}^{\infty} e^{-i 2 \pi f t} g(t) d t, k_{\mathrm{B}} T$ is the Boltzmann factor, $\epsilon_{0}$ is the dielectric permittivity of vacuum, and $\dot{\Phi}$ $=\partial \Phi(t) / \partial t$. In our simulations, $\boldsymbol{M}_{\|}$is calculated from a sum over the molecular dipole moments, as higher moments are negligible in this case. ${ }^{40}$ For the perpendicular response, on the other hand, higher moments do contribute and we thus calculate $M_{\perp}$ from an integral over the laterally averaged charge density and thereby include all higher moments. ${ }^{40}$ The volume $V$ of the water slab is defined by the average separation between the surface hydrogen atoms. This choice influences the dielectric spectra of the water slab but, in the absence of additional approximations, not the derived effective spectra for a system including water and substrate material (see the supplementary material ${ }^{43}$ ). We check our linear response method by simulations with an explicit time-dependent electric field and find excellent agreement (see the supplementary material).

Figures $1(\mathrm{~d})-1(\mathrm{~g})$ compare the simulated familiar dielectric spectrum of bulk water, featuring a static value of $\epsilon^{\prime}$ $(f \rightarrow 0)=71$ for SPC/E water ${ }^{44}$ and an absorption peak in $\epsilon^{\prime \prime}(f)$ at $f=15 \mathrm{GHz}$ (black lines), to the spectra of water slabs whose thickness is varied from $d=6.7 \mathrm{~nm}$ down to $1.1 \mathrm{~nm}$. We only consider dielectric frequencies up to $100 \mathrm{GHz}$ and thus far below the first hydrogen-bond bending mode at $1.8 \mathrm{THz}^{2,4}$ where classical MD force fields accurately reproduce experimental spectra (see Appendix A). In slabs, the dielectric function is strongly anisotropic: In the low frequency range the parallel dielectric response at the hydrophobic surface in Fig. 1(d) remains virtually unchanged for varying $d$, whereas the perpendicular response in Fig. 1(e) strongly depends on $d$, in agreement with previous static results. ${ }^{40,41}$ Compared to bulk, the absorption peak in the imaginary part in Figs. 1(d) and 1(e) shows a slight redshift for parallel fields and a pronounced blueshift in the perpendicular case with increasing confinement. Especially, the blueshift is quite dramatic and moves the peak frequency up to more than $100 \mathrm{GHz}$ for the $d=1.1 \mathrm{~nm}$ slab. The results for the hydrophilic system in Figs. 1(f) and 1(g) are similar, which shows that anisotropic red- and blueshifts are rather generic and not dependent on the specific surface chemistry.

\section{B. Spatially resolved dielectric profiles}

The slab results in Fig. 1 mix the dielectric response of the hydration and bulk-like water portions in a nonlinear fashion as we will now demonstrate. To disentangle bulk and hydration layer contributions, we spatially resolve the dielectric profile. For this, we define the correlation function $\varphi$ between the local polarization density $\boldsymbol{m}_{\alpha}(z)$ and the total polarization $\boldsymbol{M}_{\alpha}$ as

$$
\varphi_{\alpha}(t, z)=\left\langle\boldsymbol{m}_{\alpha}(0, z) \boldsymbol{M}_{\alpha}(t)\right\rangle-\left\langle\boldsymbol{m}_{\alpha}(z)\right\rangle \cdot\left\langle\boldsymbol{M}_{\alpha}\right\rangle, \quad \alpha=\|, \perp
$$

from which the local dielectric spectrum at position $z$ follows as

$$
\epsilon_{\|}(f, z)=1+L_{i f}\left(-\dot{\varphi}_{\|}\right) /\left(2 \epsilon_{0} k_{\mathrm{B}} T\right)
$$

for the parallel and

$$
\frac{\epsilon_{\perp}(f, z)-1}{\epsilon_{\perp}(f, z)}=\frac{L_{i f}\left(-\dot{\varphi}_{\perp}\right)}{\epsilon_{0} k_{\mathrm{B}} T}
$$

for the perpendicular case (the detailed derivation can be found in the supplementary material). Figures 2 and 3 compare the density profile of water with the spatially resolved dielectric profiles in the parallel and perpendicular direction for a hydrophobic surface at two frequencies $f=15 \mathrm{GHz}$ (corresponding to the bulk water absorption peak) and $f=100 \mathrm{GHz}$. The parallel absorption profile $\epsilon_{\|}^{\prime \prime}(z)$ in Fig. 2(b) is roughly proportional to the density profile for a given frequency, in agreement with previous static results. ${ }^{40,41}$ In contrast, the perpendicular profile $\epsilon_{\perp}^{\prime \prime}(z)$ in Fig. 2(c) exhibits a marked depression in the interfacial region and most noteworthy a crossing of the two curves in the second water hydration shell (corresponding to the second local density maximum in Fig. 2(a)). This crossing is connected to the blueshift of $\epsilon_{\perp}^{\prime \prime}(f)$ observed in Figs. 1(e)-1(g) and shows that it is caused by properties of the first hydration layer. To bring this out more clearly, in Figs. 2(d) and 2(e) we show frequency dependent absorption spectra in five slices of $0.6 \mathrm{~nm}$ thickness, obtained by spatially averaging the polarization correlation functions $\varphi_{\alpha}(t, z)$.
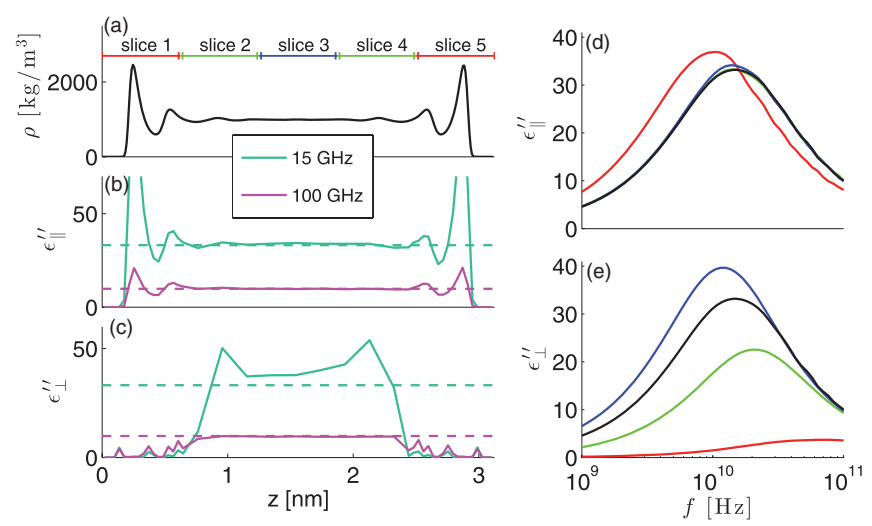

FIG. 2. Spatially resolved dielectric absorption for the hydrophobic slab with thickness $d=3.1 \mathrm{~nm}$. (a) Density profile, (b) parallel, and (c) perpendicular imaginary dielectric function at two representative frequencies $f=15 \mathrm{GHz}$ and $f=100 \mathrm{GHz}$. The bulk values are indicated by dashed lines. (d) and (e) Frequency dependent absorption averaged over five slabs of finite thickness, color-coded as indicated in the top portion of (a). The central slab (blue) is clearly bulk-like (black), while the interfacial slabs (red) show similar features as the thin water slabs in Fig. 1. 

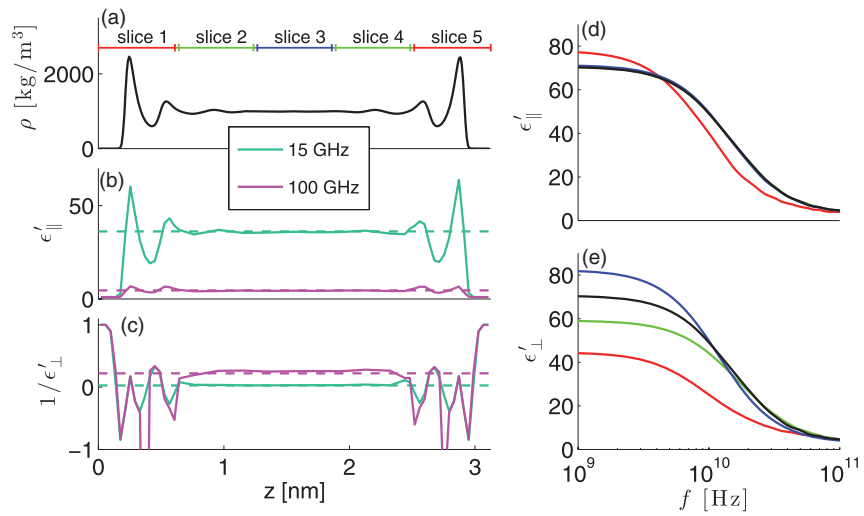

FIG. 3. Spatially resolved dielectric profile for the real part of the dielectric function for the hydrophobic surface. Note that $\epsilon_{\perp}^{\prime}(f, z)$ exhibits divergences similar to the static case ${ }^{40}$ which is why in (c) we plot the inverse $1 / \epsilon_{\perp}^{\prime}$. In the bulk region $0.7 \mathrm{~nm}<z<2.4 \mathrm{~nm}$ a resolution of $0.2 \mathrm{~nm}$ was used, while the interface was resolved in steps of $0.04 \mathrm{~nm}$ (the same resolution was used for all spatially resolved profiles).

Indeed, the interfacial slab (red lines), which fully encompasses the first hydration layer of water, shows behavior similar to the thin-slab results in Fig. 1, the second slab (green lines) shows intermediate behavior, while the central slab (blue lines) exhibits a spectrum similar to bulk (black lines). This shows that interfacial spectral features are rather localized and cross over to bulk behavior roughly in the second water hydration layer. The naive interpretation of the spectral features and, in particular, of the perpendicular blueshift would be, that the perpendicular relaxation of interfacial water is faster than in bulk; this interpretation, however, is not borne out by our microscopic analysis, as detailed below. The parallel real component in Fig. 3(b) behaves similarly to the density profile in Fig. 3(a). The perpendicular real component in Fig. 3(c) on the other hand exhibits multiple sign changes close to the interface, as found earlier in the zero-frequency limit. ${ }^{40}$

\section{Effective-medium theory for solutions of hydrated particles}

In current spectroscopic studies aimed at investigating hydration layer properties, the average dielectric absorption spectra of particle solutions are recorded, ${ }^{1-4}$ which result from a combination of solute, hydration layer, and solvent polarization as well as depolarization effects due to interfacial dielectric mismatches. ${ }^{5,45}$ Extracting hydration layer dielectric properties thus requires suitable theoretical modeling: Accordingly, we have devised a modified MGT for dilute solutions of spherical particles that accounts for tensorial dielectric effects in the hydration layer surrounding the solute particles. In our model, and as indicated in Fig. 4(a), the spherical core of radius $R_{C}$ corresponds to the actual solute particle with an internal dielectric spectrum $\epsilon_{C}(f)$ (which we assume frequency-independent, isotropic and constant in the examples shown here), the shell of outer radius $R_{S}$ models the water hydration layer with in general anisotropic dielectric spectra $\epsilon_{\perp}(f)$ and $\epsilon_{\|}(f)$. We note that existing core-shell models for particle solvation (e.g., Refs. 5, 7, 46, and 47) have thus far only considered isotropic dielectric functions for the shells around the solute particle. Finally, $R_{B}$ $=(4 \pi c / 3)^{-1 / 3}$ is the outer radius of a bulk-like solvent layer and equals approximately half the average distance between the core-shell particles in a solution of number concentration $c$ as indicated in Fig. 4(b). This solvent layer is characterized by the isotropic bulk dielectric spectrum $\epsilon_{B}(f)$ (see Appendix $\mathrm{C}$ for a detailed derivation and discussion).

Figures 4(c)-4(e) show the effective spectral absorption $\epsilon_{\text {eff }}^{\prime \prime}(f)$ for spherical particles with a core radius $R_{C}=0.36 \mathrm{~nm}$ corresponding to tetramethylurea (TMU), which is amply studied experimentally as a prototypical hydrophobic solute. ${ }^{15,48}$ In Fig. 4(c), solid lines correspond to the original MGT where shell as well as solvent regions are modeled by the simulated bulk water spectrum $\epsilon_{B}(f)$. The resultant $\epsilon_{\text {eff,orig }}^{\prime \prime}(f)$ for different particle concentrations $c_{M}$ shows no shift but just a minimal depression due to the mixing of the low-dielectric core (which models TMU and is taken to be $\left.\epsilon_{C}=1\right)$ and water. We also show a linear superposition approximation (LSA, broken lines) according to which

$$
\epsilon_{\text {eff,lsa }}^{\prime \prime}(f)=\epsilon_{\mathrm{B}}^{\prime \prime}(f)\left(R_{B}^{3}-R_{C}^{3}\right) / R_{B}^{3} .
$$

We note that depolarization effects, that are included in MGT but not in the linear superposition approximation of Eq. (7), lower the value of $\epsilon_{\text {eff,orig }}^{\prime \prime}(f)$ and could in a naive analysis of experimental data erroneously be interpreted as due to immobilized and therefore dielectrically "transparent" hydration water. Figure 4(d) presents $\epsilon_{\text {eff, iso }}^{\prime \prime}(f)$ for the isotropic approximation where $\epsilon_{\perp}^{\prime \prime}$ and $\epsilon_{\|}^{\prime \prime}$ in the hydration shell are assumed identical and equal to the simulated $\epsilon_{\|}^{\prime \prime}(f)$ in the $d=1.1 \mathrm{~nm}$ hydrophobic slab of Fig. 1(d), giving rise to a frequency shift. Finally, in Fig. 4(e), the shell is modeled as anisotropic with $\epsilon_{\|}^{\prime \prime}(f)$ and $\epsilon_{\perp}^{\prime \prime}(f)$ taken from the planar hydrophobic $d=1.1$ nm slab simulation shown in Figs. 1(d) and 1(e): The results exhibit the experimentally known redshift and a pronounced absorption depression, ${ }^{15}$ which is thus shown to be due to the unique combination of the redshift in $\epsilon_{\|}^{\prime \prime}(f)$ and an attenuation of the absorption peak in $\epsilon_{\perp}^{\prime \prime}(f)$.

Figures 4(f)-4(h) show analogous results for particles with a core radius $R_{C}=2 \mathrm{~nm}$ and $\epsilon_{C}=1$, corresponding to a solution of hydrophilic $\mathrm{C}_{12} \mathrm{E}_{5}$ micelles of similar size. ${ }^{49}$ In Fig. 4(f), we compare the original MGT (solid lines) where shell and solvent regions are bulk water-like, with the linear superposition approximation (broken lines), showing again a small but significant difference due to depolarization effects. For the isotropic approximation in Fig. 4(g), we use the hydrophilic $d=1.2 \mathrm{~nm}$ slab result for $\epsilon_{\|}^{\prime \prime}(f)$ from Fig. 1(f) as appropriate for the hydrophilic surface of $C_{12} E_{5}$ micelles; the spectra point to a strongly decreased absorption, which is due to the large core radii and the correspondingly large volume fractions achievable with micelles. The fully anisotropic calculation in Fig. 4(h) exhibits at a volume fraction $w=0.2$ a relative shift in the maximum frequency of $\Delta f_{\max } / f_{\max }=-0.12$ and in the maximum height of $\Delta \epsilon_{\text {eff,max }}^{\prime \prime} / \epsilon_{\text {eff,max }}^{\prime \prime}=-0.40$, in good agreement with experimental results $\Delta f_{\max } / f_{\max }=-0.13$ and $\Delta \epsilon_{\text {eff, max }}^{\prime \prime} / \epsilon_{\text {eff,max }}^{\prime \prime}$ $=-0.32 .{ }^{25}$ Our results show that depending on solute radius and solute concentration, depolarization effects as well as the dielectric anisotropy in the hydration layer are important for a quantitative description of experimental solution spectra. 


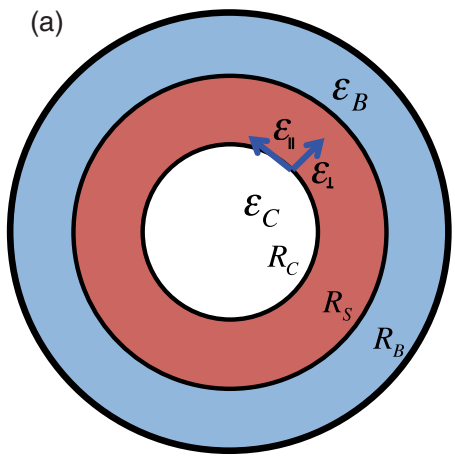

(b)
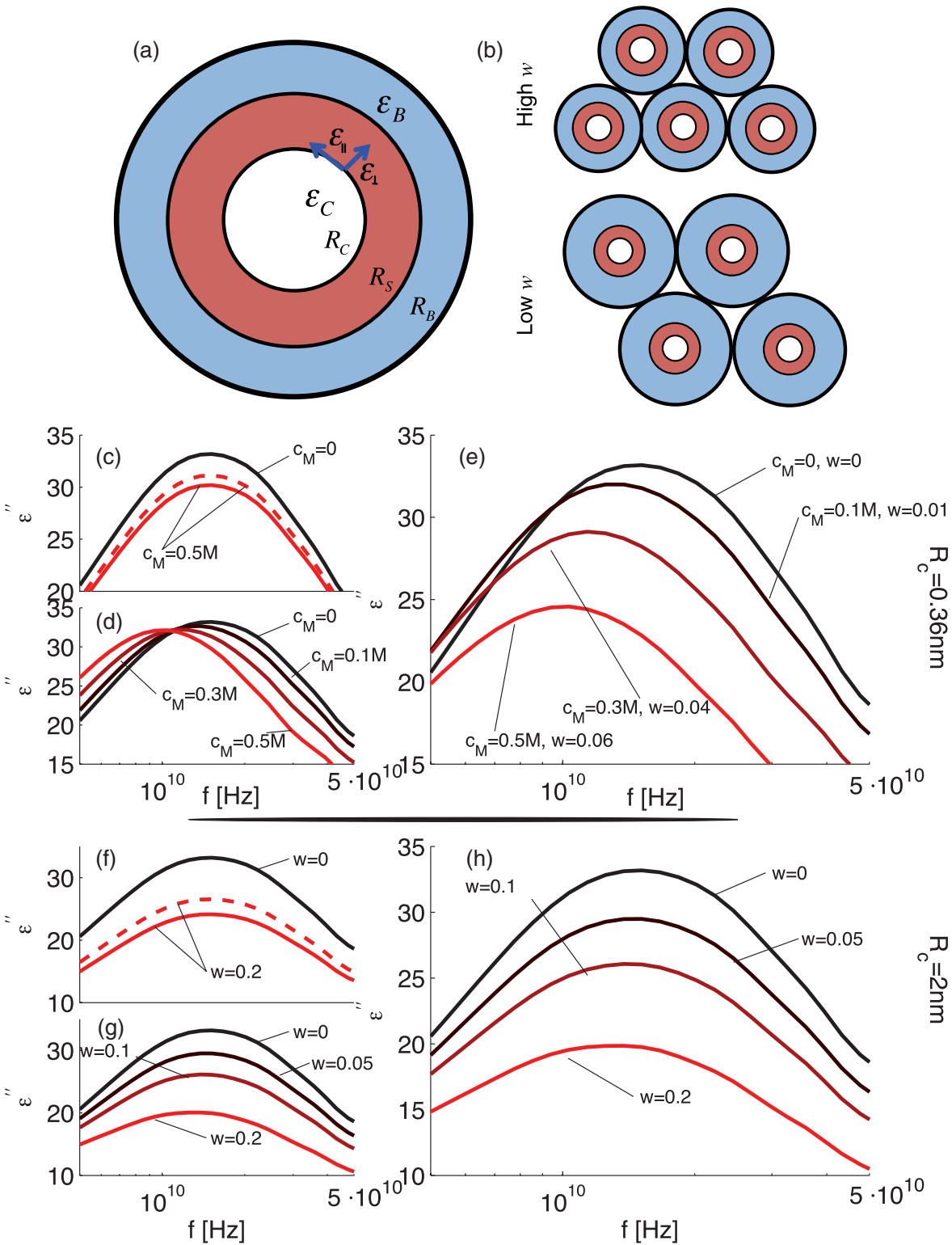

FIG. 4. Dielectric absorption $\epsilon_{\mathrm{eff}}^{\prime \prime}$ for a solution of spherical core-shell particles at various concentrations $c_{M}(\mathrm{M}=$ mol/l) or volume fractions $w$ from a modified Maxwell-Garnett theory (MGT) including our simulated tensorial interfacial dielectric spectra. (a) and (b) Schematic visualization of the MGT that consists of a core of radius $R_{C}$ representing the spherical solute particle, a shell of outer radius $R_{S}$ containing the anisotropic hydration layer, and the bulk-like solvent of radius $R_{B}$. (c)-(e) Results for core radius $R_{C}=0.36 \mathrm{~nm}$ and $\epsilon_{C}=1$, as appropriate for a tetramethylurea solution: (c) Interfacial shell and solvent entirely described by the bulk water dielectric spectrum $\epsilon_{B}(f)$, solid lines denote results from MGT, broken lines a linear superposition approximation as given by Eq. (7). (d) Shell for $R_{C}<r<R_{S}=R_{C}+d / 2$ is approximated as isotropic and described by the parallel simulated spectrum $\epsilon_{\|}(f)$ of the $d=1.1 \mathrm{~nm}$ hydrophobic slab. (e) Shell is described by full anisotropic spectra $\epsilon_{\|}(f)$ and $\epsilon_{\perp}(f)$ of the hydrophobic $d=1.1 \mathrm{~nm}$ slab. (f)-(h) Similar results for core radius $R_{C}=2 \mathrm{~nm}$ and $\epsilon_{C}=1$, appropriate for a $C_{12} E_{5}$ micellar solution: (f) Shell and solvent region modeled by bulk spectra using MGT (solid lines) or linear superposition approximation (broken lines), (g) shell spectrum assumed isotropic, and (h) fully anisotropic, modeled by the simulated spectra in the $d=1.2 \mathrm{~nm}$ hydrophilic slab. Results in (h) for relative frequency and amplitude shift of the maximum agree nearly quantitatively with experimental data. ${ }^{25}$ The black line in all figures denotes the water bulk spectrum corresponding to vanishing particle concentration.

Note that by inserting our simulation results for planar interfaces into our modified MGT we neglect possible curvature effects which for the large micellar system is not a concern but for the smaller TMU system introduces an error that will be investigated in the future.

\section{Relation to water orientational dynamics}

NMR, ${ }^{8,14}$ infrared pump-probe, ${ }^{7,9}$ and simulation studies $^{10,50}$ consistently find a retardation of the individual molecule relaxation (considering either the dipole and/or the
$\mathrm{OH}$ vector) close to various interfaces. Here, we explore the connection to our spectroscopic results. For that we define the anisotropic dipolar correlation function

$$
\eta_{\alpha}(t)=\frac{\left\langle\boldsymbol{\mu}_{\alpha}(0) \cdot \boldsymbol{\mu}_{\alpha}(t)\right\rangle}{\left\langle\boldsymbol{\mu}_{\alpha}(0) \cdot \boldsymbol{\mu}_{\alpha}(0)\right\rangle} \quad \alpha=\|, \perp,
$$

where $\boldsymbol{\mu}_{\alpha}$ denotes the dipole vector of a given water molecule projected with respect to the interface, as well as the isotropic analogue $\eta(t)=\langle\boldsymbol{\mu}(0) \boldsymbol{\mu}(t)\rangle /\langle\boldsymbol{\mu}(0) \boldsymbol{\mu}(0)\rangle$. Figure 5 confirms that the isotropic dipolar relaxation is, compared to bulk water, slowed down in all slabs. The increase of the relaxation 


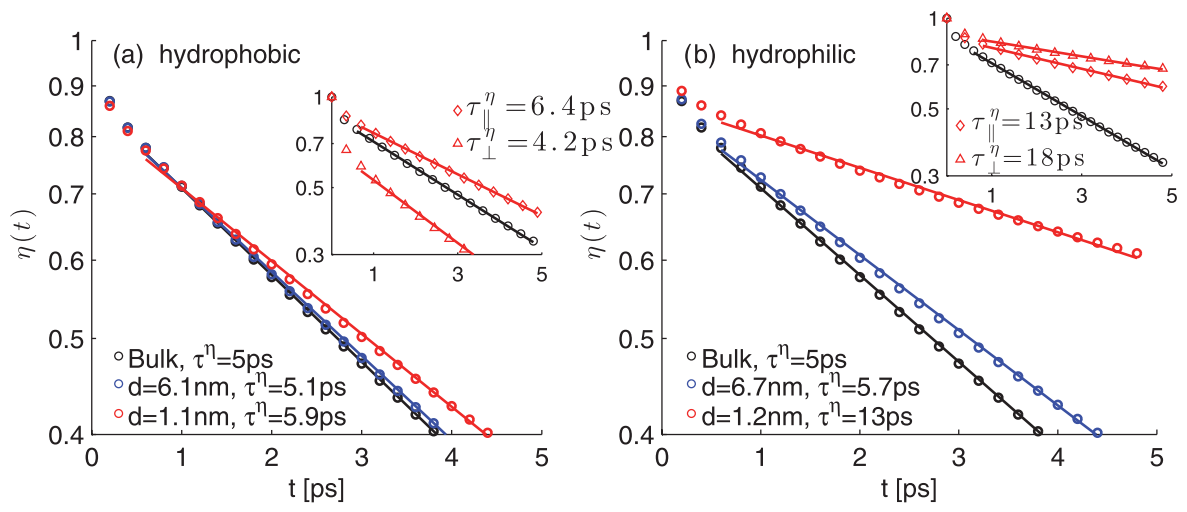

FIG. 5. Dipolar orientational correlation functions $\eta(t)$ for bulk (black), thick (blue), and thin (red) slabs at (a) hydrophobic and (b) hydrophilic surfaces. Symbols denote simulation data, lines are single-exponential fits yielding decay times as indicated. The retardation in slabs is in agreement with experiments. ${ }^{7-9,14}$ (Inset) Anisotropic correlation functions $\eta_{\perp}$ and $\eta_{\|}$in the thin slab compared with bulk.

time is strongest in the $d=1.2 \mathrm{~nm}$ hydrophilic slab and can be rationalized by numerous strong hydrogen bonds between water and polar surface groups in terms of the "extended-jump model." ${ }^{.51}$ Separation into parallel and perpendicular components (insets) demonstrates that there is no direct connection between the anisotropy observed in the dielectric spectra in Fig. 1 and the dipolar relaxation dynamics of single water molecules: In the hydrophobic $d=1.1 \mathrm{~nm}$ slab, the parallel component is slowed down and the perpendicular is accelerated compared to bulk water, whereas in the hydrophilic $d=$ $1.2 \mathrm{~nm}$ slab both components are slowed down. So the pronounced blueshift in the perpendicular dielectric spectra is, in particular at hydrophilic surfaces, not mirrored by accelerated reorientational perpendicular motion of individual water molecules in thin slabs.

To reconcile these perplexing findings, we plot in Fig. 6(a) the parallel polarization correlation function $\Phi_{\|}$in the hydrophobic slabs and compare to bulk. After an ini- tial fast decay, $\Phi_{\|}(t)$ can be fitted well with a single exponential function $A_{\|} e^{-t / \tau_{\|}}$(solid lines), where $\tau_{\|}$is the characteristic decay time. For a purely exponential correlation $\Phi_{\|}(t)$, Eq. (2) yields for $\epsilon_{\|}^{\prime \prime}(f)$ a peak frequency of $f_{\max , \|}$ $=1 /\left(2 \pi \tau_{\|}\right)$. This prediction compares very well with the maximum of the numerically calculated spectrum $\epsilon_{\|}^{\prime \prime}(f)$ in Fig. 1, as demonstrated in Fig. 6(b), and shows that the redshift in the parallel dielectric spectrum is quantitatively accounted for by the slowing down of the parallel polarization dynamics in slabs. Although the numbers do not match precisely, the slowing down of the polarization correlation function mirrors the slowed parallel molecular reorientation in the slabs as observed in Fig. 5(a). In Fig. 7(a), we plot the shorttime residual polarization correlation which is obtained by subtracting the exponential tail, defined as

$$
\Delta \Phi(t)=\Phi(t)-A e^{-t / \tau}
$$
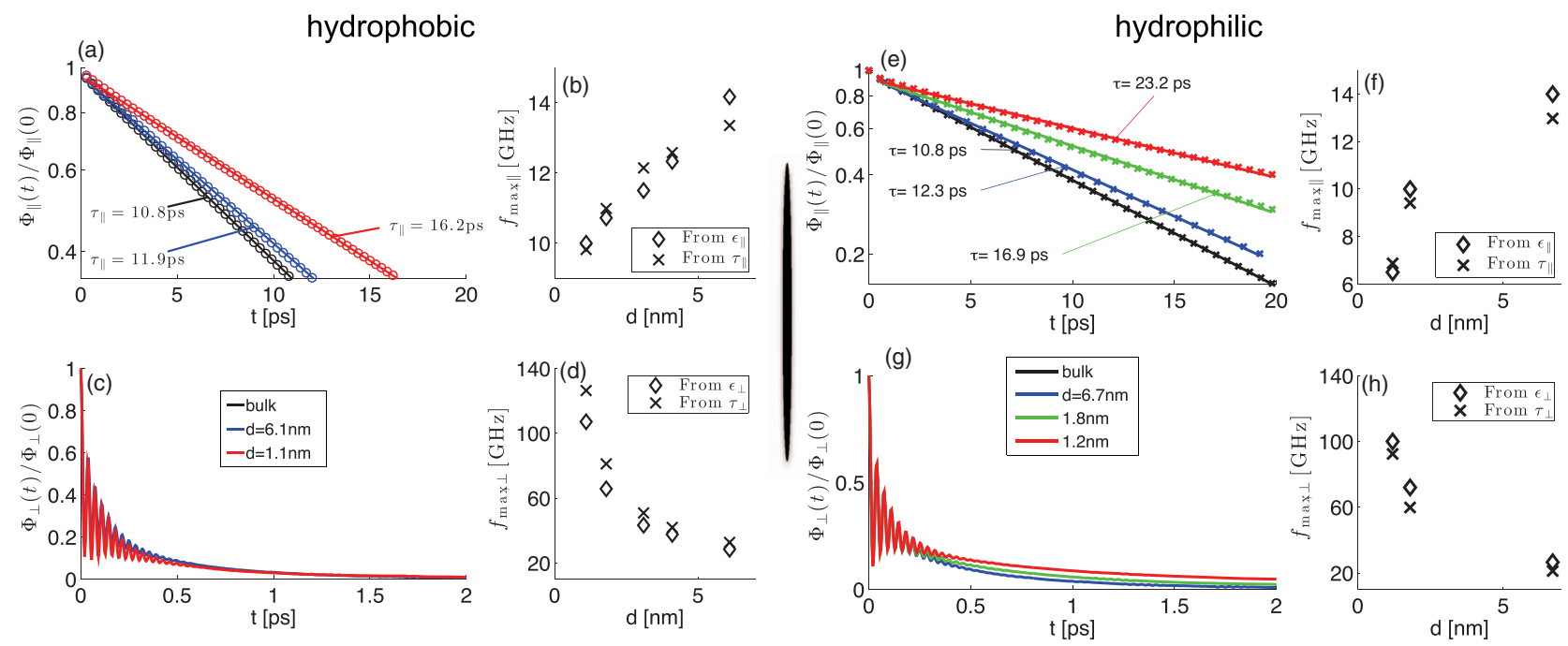

FIG. 6. (a) Parallel polarization correlation function $\Phi_{\|}(t)$ in bulk (black), thick (blue), and thin hydrophobic slabs (red) together with single exponential fits (solid lines, decay times indicated). (b) Redshift of parallel response as function of slab thickness $d$ from the maximum in $\epsilon_{\|}^{\prime \prime}(f)$ in Fig. 1 (diamonds) and via $f_{\max , \|}=1 /\left(2 \pi \tau_{\|}\right)$(crosses) using decay times $\tau_{\|}$obtained from the fits in (a). (c) Perpendicular polarization correlation function $\Phi_{\perp}(t)$. (d) Blueshift of perpendicular response for varying $d$ from the maximum in $\epsilon_{\perp}^{\prime \prime}(f)$ in Fig. 1 (diamonds) and via Eq. (13) (crosses) using decay time $\tau_{\perp}=0.145$ ps. (e) and (f) Next to a hydrophilic interface the decay of the parallel correlation function is slowed down considerably stronger than at a hydrophobic surface. (g) For the hydrophilic system, we find $\tau_{\perp} \approx 0.3 \mathrm{ps}$ where the deviation between the different slab thicknesses is around 50\% and thus larger than in the hydrophobic case. (h) Nevertheless, the predictions for the absorption maximum are in good quantitative agreement with the simulation data. 


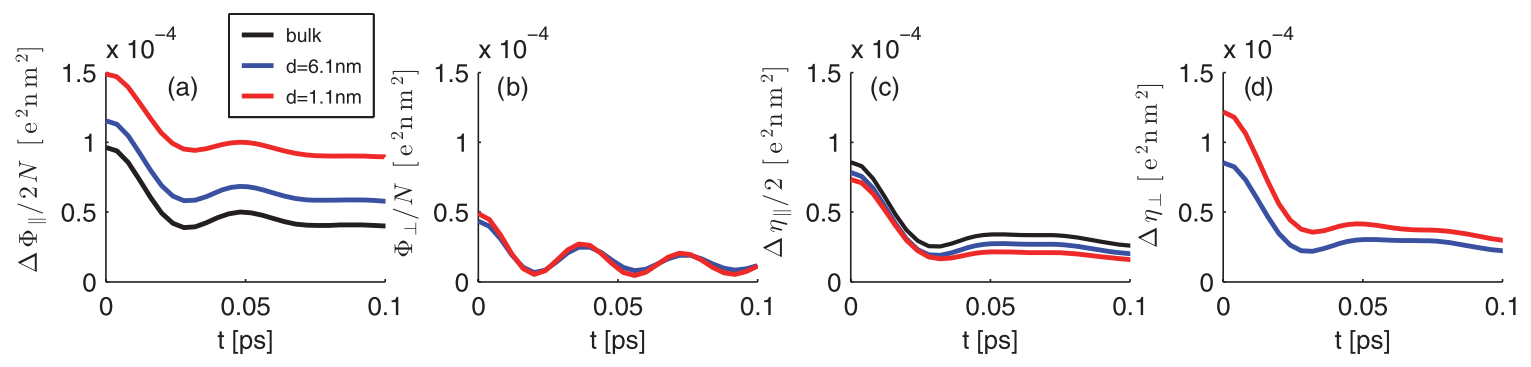

FIG. 7. (a) Short-time residual decay $\Delta \Phi(t) / 3 N$ and $\Delta \Phi_{\|}(t) / 2 N$ defined in Eqs. (9) and (10) for bulk and parallel component, respectively, normalized by the number of molecules $N$. Hydrophobic interface. (b) The oscillations in the perpendicular component $\Phi_{\perp}(t)$ are of similar absolute magnitude and thus can be attributed to the same librational oscillations as those in (a). (c) and (d) Very similar librations are further observed in the decay of the residual single-molecule orientational correlation functions as defined in Eq. (11). For a quantitative comparison and data for the hydrohpilic case see the supplementary material. All data for hydrophobic slabs.

for bulk and

$$
\Delta \Phi_{\|}(t) \equiv \Phi_{\|}(t)-A_{\|} e^{-t / \tau_{\|}}
$$

for the parallel component in slabs. We observe a fast initial decay with a single librational rebounce during the first $\sim 100 \mathrm{fs}$, in agreement with earlier experimental and simulation studies. ${ }^{33,52}$

The perpendicular polarization $\Phi_{\perp}(t)$ in Fig. 6(c) is dramatically different and displays pronounced oscillations. Those oscillations were for a dipolar model fluid rationalized by a "dipolaron" mode. ${ }^{42}$ Here, we provide an alternative explanation and argue instead that $\Phi_{\perp}(t)$ is dominated by librational effects: This is demonstrated by the fact that the initial decay of $\Phi_{\perp}(t)$ in Fig. 7(b) is quite similar in magnitude and shape to the residual parallel and bulk polarization correlations $\Delta \Phi_{\|}(t)$ in Fig. 7(a). Incidentally, very similar features are also seen in the single molecule dipolar correlation functions $\eta(t)$ at short times. To demonstrate this, we define the non-normalized single-molecule orientational correlation functions with the exponential tail subtracted

$$
\begin{aligned}
\Delta \eta(t) & =\langle\boldsymbol{\mu}(0) \cdot \boldsymbol{\mu}(t)\rangle-A^{\eta} e^{-t / \tau^{\eta}}, \\
\Delta \eta_{\|}(t) & =\left\langle\boldsymbol{\mu}_{\|}(0) \cdot \boldsymbol{\mu}_{\|}(t)\right\rangle-A_{\|}^{\eta} e^{-t / \tau_{\|}^{\eta},} \\
\Delta \eta_{\perp}(t) & =\left\langle\mu_{\perp}(0) \mu_{\perp}(t)\right\rangle-A_{\perp}^{\eta} e^{-t / \tau_{\perp}^{\eta},}
\end{aligned}
$$

where the parameters $A$ and $\tau$ are obtained from fits such as those displayed in Fig. 5. As shown in Figs. 7(c) and 7(d), all features - including the seemingly very large oscillations in $\Phi_{\perp}-$ are of comparable absolute magnitude and period. This strongly supports our interpretation that they all correspond to the same physical phenomenon, with the only difference that the damping is significantly weaker in the collective perpendicular polarization correlation function than in the other cases. In line with earlier studies, ${ }^{33,52}$ we attribute the rebounces to librational motions which lead to spectral features above $1 \mathrm{THz}$ and are thus not relevant for the present work. In essence, $\Phi_{\perp}(t)$ reflects the librational motion of the water molecules in an unobstructed fashion, whereas librational effects in $\Phi_{\|}(t)$ are overshadowed by an additional exponentially decaying slow contribution (which is dampened in $\Phi_{\perp}(t)$ because of the different dielectric boundary condition).

A second striking feature of Fig. 6(c) is that the decay of the correlation function seems to be independent of the slab thickness. We define the perpendicular correlation time as

$$
\tau_{\perp}=\int_{0}^{\infty} \Phi_{\perp}(t) / \Phi_{\perp}(0) d t
$$

and obtain $\tau_{\perp}=0.145 \pm 0.05 \mathrm{ps}$ for the hydrophobic surfaces and all three different slab thicknesses. How does this insensitivity of the perpendicular polarization dynamics harmonize with the pronounced absorption blueshift in $\epsilon_{\perp}^{\prime \prime}(f)$ for decreasing slab thickness displayed in Figs. 1(e) and 1(g)? For an exponentially decaying function $\Phi_{\perp}(t)$ with decay time $\tau_{\perp}$, Eq. (3) predicts a maximum in $\epsilon_{\perp}^{\prime \prime}(f)$ at a frequency (see Appendix D)

$$
f_{\max , \perp}=\frac{1}{\epsilon_{\perp}^{\prime}(f=0)} \frac{1}{2 \pi \tau_{\perp}} .
$$

This is in quantitative agreement with the maximum in $\epsilon_{\perp}^{\prime \prime}(f)$ inferred directly from the simulation data in Fig. 1, as shown in Figs. 6(d) and 6(h) for the hydrophobic and hydrophilic surfaces, respectively. Incidentally, Eq. (13) also correctly predicts $f_{\max , \perp}=15.5 \mathrm{GHz}$ in the bulk limit, when the SPC/E static bulk value $\epsilon_{\perp}^{\prime}(f=0)=71$ is used in conjunction with the correlation time $\tau_{\perp}=0.145 \mathrm{ps}$. Thus, the salient blueshift in the perpendicular dielectric absorption is related to a decrease of the static perpendicular dielectric constant $\epsilon_{\perp}^{\prime}(f=0)$ and not to an acceleration of the water reorientation dynamics. This finding serves as an example that the relation between dielectric features and molecular dynamics can be quite subtle and correct molecular interpretation of experimental data, therefore, requires correct theoretical modeling.

\section{CONCLUSION}

Using classical MD simulations of water at hydrophobic and hydrophilic surfaces, we show that the dielectric absorption in hydration layers is highly anisotropic: While the imaginary part of the dielectric function describing the response to surface-parallel electric fields shows only a weak attenuation and redshift of the absorption peak compared to bulk water, the response function for perpendicular fields is strongly attenuated and blueshifted towards higher frequencies $(>100 \mathrm{GHz})$. From spatially resolved calculations of the dielectric spectra, this anisotropy is traced to the localized water region within the first hydration shell. Only mild differences between hydrophilic and hydrophobic surfaces are found. 
Incorporating our simulated spectra into a modified MaxwellGarnett theory that accounts for the anisotropic absorption in a hydration shell around spherical particles, the absorption spectrum of a solution is shown to exhibit the experimentally well-known redshift and absorption attenuation. Nearly quantitative agreement with experimental data for micellar solutions is obtained. Thus, the spectral dielectric absorption features of particle solutions result from an intricate combination of anisotropic hydration layer properties: The parallel component gives rise to the redshift, the perpendicular component in conjunction with the solute contribution is responsible for the absorption attenuation.

Interestingly, the perpendicular blueshift is not related to an acceleration of single water molecule reorientation, but rather shown to be due to dielectric boundary effects: The relation between the polarization correlation function and the dielectric spectrum involves in addition to the correlation time also the static dielectric constant in the perpendicular case. We conclude that the correct molecular interpretation of dielectric absorption data needs correct theoretical modeling. Our modified Maxwell-Garnett theory that includes anisotropy in the hydration shell by using simulated spectra in planar slabs should be viewed as a first step in that direction.

\section{ACKNOWLEDGMENTS}

We thank Forschungszentrum Jülich for computing time under Project HMU16, the Leibniz-Rechenzentrum under Project pr28xe and the Deutsche Forschungemeinschaft for financial support under Project GE2214/1.

\section{APPENDIX A: THE SPECTRUM OF BULK WATER}

In Fig. 8, we compare the dielectric spectrum obtained from simulations of bulk water with experimental data from Ref. 2 and find satisfying agreement, especially for the position of the absorption peak. The simulations use 895 water molecules in a box of $3 \times 3 \times 3 \mathrm{~nm}^{3}$ at a temperature of $300 \mathrm{~K}$, a pressure of 1 bar and were run for $350 \mathrm{~ns}$.

\section{APPENDIX B: DERIVATION OF EQS. (2)-(6) OF THE MAIN TEXT}

Our derivation extends that given in Ref. 38 taking into account a non-zero average polarization as well as adding the possibility to consider individual slices (not only the system as a whole). We will in the following derive only Eqs. (5) and (6) of the main text. Equations (2) and (3) can then trivially be obtained by extending the slice to include the entire slab. We start by defining the pulse response function $f_{p}(t)$ which describes the polarization of the system at time $t$ induced by an electric field pulse at $t=0$. An externally applied time-dependent electric field $\Delta \boldsymbol{E}(t)$ thus gives the induced polarization density $\Delta \boldsymbol{m}$ at time $t$ as

$$
\Delta \boldsymbol{m}(t, z)=\chi_{s}(z) \epsilon_{0} \int_{-\infty}^{t} \Delta \boldsymbol{E}\left(t^{*}, z\right) f_{p}\left(t-t^{*}, z\right) d t^{*},
$$

where $\chi_{s}$ is the static susceptibility. For clarity, we will in the following omit the $z$-dependence. For a sinusoidal electric

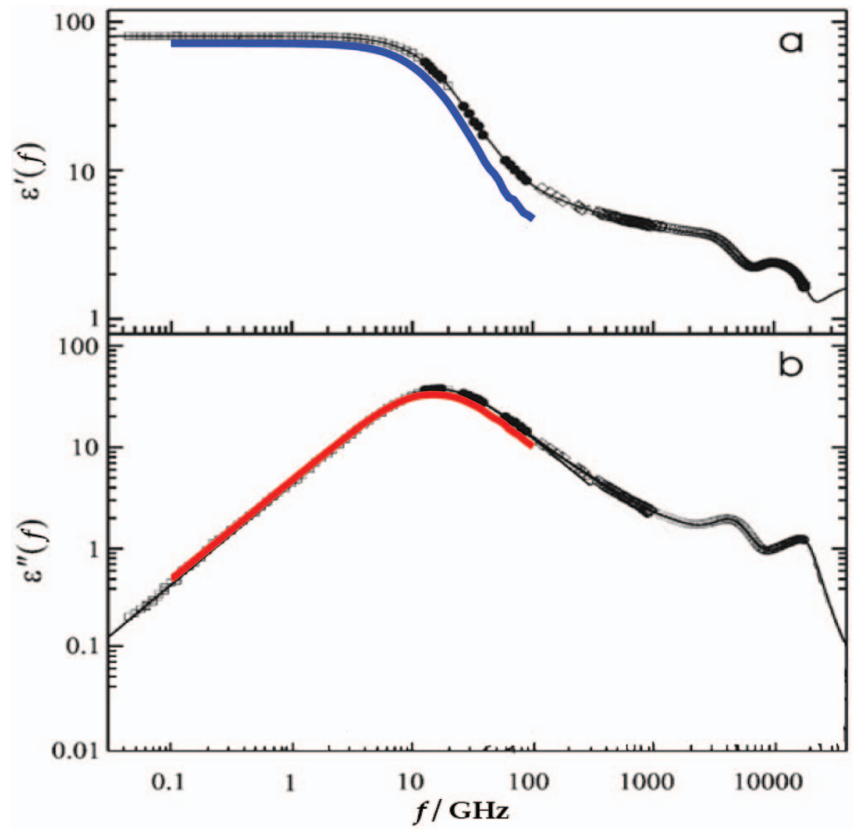

FIG. 8. Comparison of the real part (top) and the imaginary part (bottom) of the dielectric spectrum of bulk water between experiments ${ }^{2}$ and our MD simulations (colored lines). The position of the absorption peak is well captured by our simulations.

field with frequency $f$,

$$
\Delta \boldsymbol{E}\left(t^{*}\right)=\boldsymbol{E}_{0} \cos \left(2 \pi f t^{*}\right),
$$

we can write

$$
\Delta \boldsymbol{m}(t)=\boldsymbol{E}_{0} \chi_{s} \epsilon_{0} \int_{-\infty}^{t} \cos \left(2 \pi f t^{*}\right) f_{p}\left(t-t^{*}\right) d t^{*} .
$$

Substituting $t-t^{*}=t^{\prime}$, we get

$$
\begin{aligned}
\Delta \boldsymbol{m}(t)= & \boldsymbol{E}_{0} \chi_{s} \epsilon_{0} \int_{0}^{\infty} \cos \left(2 \pi f\left(t-t^{\prime}\right)\right) f_{p}\left(t^{\prime}\right) d t^{\prime}, \\
= & \boldsymbol{E}_{0} \cos (2 \pi f t) \underbrace{\int_{0}^{\infty} \chi_{s} \cos \left(2 \pi f t^{\prime}\right) f_{p}\left(t^{\prime}\right) d t^{\prime}}_{\chi^{\prime}} \\
& +\boldsymbol{E}_{0} \sin (2 \pi f t) \underbrace{\int_{0}^{\infty} \chi_{s} \sin \left(2 \pi f t^{\prime}\right) f_{p}\left(t^{\prime}\right) d t^{\prime}}_{\chi^{\prime \prime}} .
\end{aligned}
$$

Introducing the complex susceptibility and the complex electric field

$$
\begin{gathered}
\chi=\chi^{\prime}-i \chi^{\prime \prime}, \\
\hat{\boldsymbol{E}}(t)=\boldsymbol{E}_{0} e^{i 2 \pi f t},
\end{gathered}
$$

and writing

$$
\begin{aligned}
\Delta \hat{\boldsymbol{m}}(t)= & \chi \hat{\boldsymbol{E}}(t), \\
= & \chi^{\prime} \boldsymbol{E}_{0}(\cos (2 \pi f t)+i \sin (2 \pi f t)) \\
& -i \chi^{\prime \prime} \boldsymbol{E}_{0}(\cos (2 \pi f t)+i \sin (2 \pi f t)), \\
= & \chi^{\prime} \boldsymbol{E}_{0} \cos (2 \pi f t)+\chi^{\prime \prime} \boldsymbol{E}_{0} \sin (2 \pi f t) \\
& +i \chi^{\prime} \boldsymbol{E}_{0} \sin (2 \pi f t)-i \chi^{\prime \prime} \boldsymbol{E}_{0} \cos (2 \pi f t)
\end{aligned}
$$


with $\Delta \boldsymbol{m}=\operatorname{Re}(\Delta \hat{\boldsymbol{m}})$ motivates the definition of $\chi^{\prime}$ and $\chi^{\prime \prime}$ in Eq. (B5). Now we substitute $\chi^{\prime}$ and $\chi^{\prime \prime}$ from Eqs. (B5) into (B6) to obtain

$$
\begin{aligned}
\chi= & \chi_{s}\left[\int_{0}^{\infty} \cos \left(2 \pi f t^{\prime}\right) f_{p}\left(t^{\prime}\right) d t^{\prime}\right. \\
& \left.-i \int_{0}^{\infty} \sin \left(2 \pi f t^{\prime}\right) f_{p}\left(t^{\prime}\right) d t^{\prime}\right] \\
= & \chi_{s} \int_{0}^{\infty} e^{-i 2 \pi f t^{\prime}} f_{p}\left(t^{\prime}\right) d t^{\prime} \\
= & \chi_{s} L_{i f}\left(f_{p}\right)
\end{aligned}
$$

where $L_{i f}$ is the Laplace transform as in the main text.

We now turn to the statistical mechanics description of our system. When a small external field $\boldsymbol{F}$ is applied, the total energy of the system at time $t$ shall be given by $\boldsymbol{F}(t) \boldsymbol{M}(t){ }^{40,41}$ For any time-dependent observable $\boldsymbol{A}(t)$ the average value in the perturbed case is given by (cf. Ref. 38, p. 145),

$$
\overline{\boldsymbol{A}}(t)=\langle\boldsymbol{A}\rangle+\frac{1}{k_{B} T} \int_{-\infty}^{t} d t^{\prime} \boldsymbol{F}\left(t^{\prime}\right)\left\langle\boldsymbol{A}(t) \dot{\boldsymbol{M}}\left(t^{\prime}\right)\right\rangle .
$$

Here, the overbar means the value of the macroscopic quantity in the perturbed system and \langle\rangle denotes an average over phase space trajectories in the equilibrium, unperturbed system as in the main text. We derive the parallel component, i.e., Eq. (5) of the main text. Here $\boldsymbol{F}_{\|}=\Delta \boldsymbol{E}_{\|}^{40}$ and we consider only the resulting parallel system dipole $\boldsymbol{M}_{\|}$. We choose $\boldsymbol{A}=\boldsymbol{m}_{\|}(t)$ where we recall that $\boldsymbol{m}_{\|}$is the instantaneous polarization density of an infinitesimally thin horizontal slice. Due to isotropy in the plane, the fluctuations in $x$ - and $y$-direction are uncorrelated and thus the tensor $\left\langle\boldsymbol{m}_{\|} \dot{\boldsymbol{M}}_{\|}\right\rangle$is diagonal and can be substituted by $1 / 2\left\langle\boldsymbol{m}_{\|} \cdot \dot{\boldsymbol{M}}_{\|}\right\rangle$where $\cdot$ denotes the scalar product. We obtain

$$
\overline{\boldsymbol{m}_{\|}}(t)-\left\langle\boldsymbol{m}_{\|}\right\rangle=\frac{1}{2 k_{B} T} \int_{-\infty}^{t} d t^{\prime} \Delta \boldsymbol{E}_{\|}\left(t^{\prime}\right)\left\langle\boldsymbol{m}_{\|}(t) \cdot \dot{\boldsymbol{M}}\left(t^{\prime}\right)\right\rangle .
$$

The left-hand side of Eq. (B15) represents the induced polarization density $\Delta \boldsymbol{m}_{\|}(t)$. Comparing Eqs. (B1) and (B15), we find

$$
\begin{aligned}
\chi_{s} \epsilon_{0} f_{p}\left(t-t^{\prime}\right) & =\frac{1}{2 k_{B} T}\left\langle\boldsymbol{m}_{\|}(t) \cdot \dot{\boldsymbol{M}}_{\|}\left(t^{\prime}\right)\right\rangle, \\
& =-\frac{1}{2 k_{B} T}\left\langle\boldsymbol{m}_{\|}\left(t^{\prime}\right) \cdot \dot{\boldsymbol{M}}_{\|}(t)\right\rangle,
\end{aligned}
$$

where in the last step we have used the fact that $\left\langle\boldsymbol{m}_{\|}(t)\right.$. $\left.\dot{\boldsymbol{M}}_{\|}\left(t^{\prime}\right)\right\rangle$ is anti-symmetric in time. Due to statistical averaging Eq. (B17) merely depends on the difference $t-t^{\prime}$ and not on $t$ and $t^{\prime}$ separately. This allows us to substitute $t-t^{\prime} \rightarrow t$ to obtain

$$
\chi_{s} \epsilon_{0} f_{p}(t)=-\frac{1}{2 k_{B} T}\left\langle\boldsymbol{m}_{\|}(0) \cdot \dot{\boldsymbol{M}}_{\|}(t)\right\rangle
$$

whose Laplace transform is

$$
\chi_{s} \epsilon_{0} L_{i f}\left[f_{p}(t)\right]=-\frac{1}{2 k_{B} T} L_{i f}\left[\left\langle\boldsymbol{m}_{\|}(0) \cdot \dot{\boldsymbol{M}}_{\|}(t)\right\rangle\right] .
$$

Substituting Eq. (B13) leads to

$$
\chi=\frac{1}{2 k_{B} T \epsilon_{0}} L_{i f}\left[-\left\langle\boldsymbol{m}_{\|}(0) \cdot \dot{\boldsymbol{M}}_{\|}(t)\right\rangle\right] .
$$

Using the definition of the correlation function

$$
\varphi_{\|}(t)=\left\langle\boldsymbol{m}_{\|}(0) \cdot \boldsymbol{M}_{\|}(t)\right\rangle-\left\langle\boldsymbol{m}_{\|}(0)\right\rangle \cdot\left\langle\boldsymbol{M}_{\|}(0)\right\rangle
$$

and the relation $\epsilon_{\|}=\chi+1$ leads to Eq. (5) of the main text.

We note that Eq. (B20) only contains the time derivative of the correlation function. Accordingly, one could use the alternative definition

$$
\varphi_{\|}^{*}(t)=\left\langle\boldsymbol{m}_{\|}(0) \cdot \boldsymbol{M}_{\|}(t)\right\rangle
$$

and obtain the same result. In order to avoid taking numerical derivatives, however, we evaluate the Laplace transforms using the well-known relation

$$
L_{i f}[-\dot{\varphi}(t)]=\varphi(0)-i 2 \pi f L_{i f}[\varphi(t)],
$$

which is much easier to evaluate when $\varphi \rightarrow 0$ for $t \rightarrow \infty$, since then the correlation functions can be cut at some finite $t$. This motivates us to prefer the definition in Eq. (B21) over that in Eq. (B22).

In the perpendicular case $F_{\perp}=D_{\perp} / \epsilon_{0}=\Delta E_{\perp} \epsilon_{\perp}{ }^{40}$ and the same procedure as above then leads to Eq. (6) of the main text.

\section{APPENDIX C: ANISOTROPIC MAXWELL-GARNETT THEORY}

In order to calculate the dielectric spectrum $\epsilon_{\text {eff }}$ of a coreshell particle as depicted in Fig. 4(a), we focus on the imaginary component and consider the amount of energy $W_{\mathrm{abs}}$ that is absorbed by the system when a time-dependent electric potential of the form

$$
\phi(z)=-E_{\mathrm{ext}}(t) z=-E_{0} e^{i 2 \pi f t} z
$$

is fixed on the outer boundary defined by the sphere radius $R_{B}$. The total amount of energy absorbed by an equivalent spherical particle with radius $R_{B}$ having a spatially homogeneous dielectric spectrum $\epsilon_{\mathrm{eff}}^{\prime \prime}$ is given by (see Ref. 38, p. 14),

$$
W_{\mathrm{abs}, \mathrm{hom}}=\frac{4}{3} \pi R_{B}^{3} \pi \epsilon_{0} \epsilon_{\mathrm{eff}}^{\prime \prime}(f) E_{0}^{2},
$$

since the electric field is homogeneous throughout the sphere in this case. This relation is used as a definition of an effective, isotropic dielectric function $\epsilon_{\mathrm{eff}}^{\prime \prime}$ for systems in which the actual dielectric spectrum is anisotropic since $W_{\text {abs }}$ is always well defined, physically meaningful and measurable.

In order to calculate $W_{\text {abs }}$ using the core-shell model, we denote the electrical potential as $\phi_{C}$ inside the core $\left(r<R_{C}\right)$, as $\phi_{S}$ inside the shell $\left(R_{C}<r<R_{S}\right)$, and as $\phi_{B}$ in the outer water layer $\left(R_{S}<r<R_{B}\right)$. Inside their respective regions, all potentials satisfy Laplace's equation. Exploiting the axisymmetry of the problem and using spherical coordinates $(r, \theta, \varphi)$ the general solutions are given by

$$
\begin{aligned}
& \phi_{C}(r, \theta)=\sum_{n=0}^{\infty}\left(A_{n} r^{n}+\frac{B_{n}}{r^{n+1}}\right) P_{n}(\cos \theta), \\
& \phi_{S}(r, \theta)=\sum_{n=0}^{\infty}\left(G_{n} r^{n}+\frac{H_{n}}{r^{n+1}}\right) P_{n}(\cos \theta),
\end{aligned}
$$




$$
\phi_{B}(r, \theta)=\sum_{n=0}^{\infty}\left(J_{n} r^{n}+\frac{L_{n}}{r^{n+1}}\right) P_{n}(\cos \theta)
$$

with $P_{n}$ denoting the Legendre polynomial of order $n$. Since $\phi_{C}$ must be finite for $r \rightarrow 0$, it follows that $B_{n}=0$ for all $n$. The five boundary conditions are

$$
\begin{gathered}
\phi_{C}=\phi_{S} \quad \text { for } r=R_{C}, \\
\epsilon_{C} \frac{d \phi_{C}}{d r}=\epsilon_{\perp} \frac{d \phi_{S}}{d r} \quad \text { for } r=R_{C}, \\
\phi_{S}=\phi_{B} \quad \text { for } r=R_{S}, \\
\epsilon_{\perp} \frac{d \phi_{S}}{d r}=\epsilon_{B} \frac{d \phi_{B}}{d r} \quad \text { for } r=R_{S}, \\
\phi_{B}=-E_{\mathrm{ext}}(t) r \cos (\theta) \quad \text { for } r=R_{B} .
\end{gathered}
$$

With the ansatz of Eqs. (C3) through (C5) we obtain linear systems of equations for $A_{n}, G_{n}, H_{n}, J_{n}$, and $L_{n}$ which can be solved separately for each $n$. Note that the coefficients $A_{n}-L_{n}$ depend on time through the fifth boundary condition Eq. (C10). No non-trivial solution exists in general for $n \neq 1$ (although for specific combinations of $R_{C}, R_{S}, R_{B}, \epsilon_{C}, \epsilon_{\perp}$, and $\epsilon_{B}$ additional resonant solutions may exist). The solution for $n=1$ reads

$$
\begin{gathered}
A_{1}(t)=-9 e^{i 2 \pi f t} E_{0} R_{B}^{3} R_{S}^{3} \epsilon_{B} \epsilon_{\perp} / D \\
G_{1}(t)=-3 e^{i 2 \pi f t} E_{0} R_{B}^{3} R_{S}^{3} \epsilon_{B}\left(\epsilon_{C}+2 \epsilon_{\perp}\right) / D \\
H_{1}(t)=-3 e^{i 2 \pi f t} E_{0} R_{C}^{3} R_{B}^{3} R_{S}^{3} \epsilon_{B}\left(-\epsilon_{C}+\epsilon_{\perp}\right) / D \\
J_{1}(t)=e^{i 2 \pi f t} E_{0} R_{B}^{3}\left(2 R_{C}^{3}\left(\epsilon_{B}-\epsilon_{\perp}\right)\left(\epsilon_{C}-\epsilon_{\perp}\right)\right. \\
\left.-R_{S}^{3}\left(2 \epsilon_{B}+\epsilon_{\perp}\right)\left(\epsilon_{C}+2 \epsilon_{\perp}\right)\right) / D \\
L_{1}(t)=-e^{i 2 \pi f t} E_{0} R_{B}^{3} R_{S}^{3}\left(-R_{C}^{3}\left(\epsilon_{C}-\epsilon_{\perp}\right)\left(\epsilon_{B}+2 \epsilon_{\perp}\right)\right. \\
\left.+R_{S}^{3}\left(\epsilon_{B}-\epsilon_{\perp}\right)\left(\epsilon_{C}+2 \epsilon_{\perp}\right)\right) / D
\end{gathered}
$$

where the common denominator is given by

$$
\begin{aligned}
D= & R_{S}^{3}\left(\epsilon_{C}+2 \epsilon_{\perp}\right)\left(R_{S}^{3}\left(\epsilon_{B}-\epsilon_{\perp}\right)+R_{B}^{3}\left(2 \epsilon_{B}+\epsilon_{\perp}\right)\right) \\
& -R_{C}^{3}\left(\epsilon_{C}-\epsilon_{\perp}\right)\left(2 R_{B}^{3}\left(\epsilon_{B}-\epsilon_{\perp}\right)+R_{S}^{3}\left(\epsilon_{B}+2 \epsilon_{\perp}\right)\right) .
\end{aligned}
$$

Now that we have computed the potential and thus the electric field at any point inside the particle, we proceed to split the electric field into radial and tangential components $E_{r}(t)$ and
$E_{\theta}(t)$, respectively,

$$
\begin{aligned}
\mathbf{E}(t) & =\left(\mathbf{E}(t) \cdot \mathbf{e}_{r}\right) \mathbf{e}_{r}+\left(\mathbf{E}(t) \cdot \mathbf{e}_{\theta}\right) \mathbf{e}_{\theta} \\
& =E_{r}(t) \mathbf{e}_{r}+E_{\theta}(t) \mathbf{e}_{\theta} \\
& =-\frac{d \phi(t)}{d r} \mathbf{e}_{r}-\frac{1}{r} \frac{d \phi(t)}{d \theta} \mathbf{e}_{\theta} .
\end{aligned}
$$

The absorbed energy per period and unit volume at any point in the shell is then calculated as

$$
\begin{aligned}
w_{\mathrm{abs}, \mathrm{S}}= & \int_{t=0}^{t=T} \operatorname{Re}[\mathbf{E}(t)] \operatorname{Re}[d \mathbf{D}(t)] \\
= & \int_{0}^{T} \operatorname{Re}[\mathbf{E}(t)] \operatorname{Re}\left[\frac{d \mathbf{D}(t)}{d t}\right] d t \\
= & \int_{0}^{T} \operatorname{Re}\left[E_{r}(t)\right] \operatorname{Re}\left[\frac{\epsilon_{\perp} d E_{r}(t)}{d t}\right] \\
& +\operatorname{Re}\left[E_{\theta}(t)\right] \operatorname{Re}\left[\frac{\epsilon_{\|} d E_{\theta}(t)}{d t}\right] d t,
\end{aligned}
$$

where $T=1 / f$ is the duration of one period, and Re denotes the real part. In the outer bulk layer, the dielectric function is isotropic and resulting expression reads

$$
\begin{aligned}
w_{\mathrm{abs}, \mathrm{B}}= & \int_{0}^{T} \operatorname{Re}\left[E_{r}(t)\right] \operatorname{Re}\left[\frac{\epsilon_{B} d E_{r}(t)}{d t}\right] \\
& +\operatorname{Re}\left[E_{\theta}(t)\right] \operatorname{Re}\left[\frac{\epsilon_{B} d E_{\theta}(t)}{d t}\right] d t .
\end{aligned}
$$

The total amount of energy absorbed inside the particle is

$$
W_{\mathrm{abs}}=\int_{\text {shell }} w_{\mathrm{abs}, \mathrm{S}} d V+\int_{\text {bulklayer }} w_{\mathrm{abs}, \mathrm{B}} d V,
$$

where the integration only extends over the volume of the anisotropic shell and the outer layer of bulk water since the core in our approximation does not absorb any energy in the considered frequency range $\left(\epsilon_{C}^{\prime \prime}=0\right)$. Equating Eqs. (C2) and (C20) yields the desired value for $\epsilon_{\mathrm{eff}}^{\prime \prime}$. By identifying the dielectric properties of the spherical shell with the spectra of the planar water slabs from our simulations we neglect curvature effects which for large radii (corresponding to the micellar system shown in Fig. 4(h) of the main text) is a good approximation leading to quantitative agreement with experimental data.

Finally, we mention two limiting cases. First, for $R_{C}$ $\rightarrow 0$ and $R_{B}=R_{S}$, we find

$$
\epsilon_{\mathrm{eff}}^{\prime \prime}=\frac{1}{3}\left(2 \epsilon_{\|}^{\prime \prime}+\epsilon_{\perp}^{\prime \prime}\right)
$$

which reflects the fact that for a solid sphere made of an anisotropic dielectric material in a homogeneous electric field the internal field samples all three spatial directions equally and thus the effective dielectric function is simply a weighted average.

Next, we consider the limit in which the shell dielectric properties are isotropic and identical to bulk water $\epsilon_{\|}=\epsilon_{\perp}$ $=\epsilon_{B}$. Such a system corresponds to the classical MaxwellGarnett scenario which predicts the following, complex 
effective dielectric function (Eq. (11) in Ref. 5),

$$
\epsilon_{\mathrm{MG}}=\epsilon_{B} \frac{2 \epsilon_{B}+\epsilon_{C}-2\left(\epsilon_{B}-\epsilon_{C}\right) R_{C}^{3} / R_{B}^{3}}{2 \epsilon_{B}+\epsilon_{C}+\left(\epsilon_{B}-\epsilon_{C}\right) R_{C}^{3} / R_{B}^{3}}
$$

with the imaginary part

$$
\begin{aligned}
\epsilon_{\mathrm{MG}}^{\prime \prime}= & \left(-2 R_{C}^{6} \epsilon_{B}^{\prime \prime}\left(\left(\epsilon_{B}^{\prime}-\epsilon_{C}^{\prime}\right)^{2}+\left(\epsilon_{B}^{\prime \prime}-\epsilon_{C}^{\prime \prime}\right)^{2}\right)\right. \\
& +R_{B}^{3} R_{C}^{3}\left(\epsilon_{B}^{\prime \prime}\left(-2\left(\epsilon_{B}^{2}+\epsilon_{B}^{\prime \prime 2}\right)-8 \epsilon_{B}^{\prime} \epsilon_{C}^{\prime}+\epsilon_{C}^{\prime 2}\right)\right. \\
& \left.+\left(9 \epsilon_{B}^{\prime 2}+\epsilon_{B}^{\prime \prime 2}\right) \epsilon_{C}^{\prime \prime}+\epsilon_{B}^{\prime \prime} \epsilon_{C}^{\prime \prime 2}\right) \\
& \left.+R_{B}^{6} \epsilon_{B}^{\prime \prime}\left(\left(2 \epsilon_{B}^{\prime}+\epsilon_{C}^{\prime}\right)^{2}+\left(2 \epsilon_{B}^{\prime \prime}+\epsilon_{C}^{\prime \prime}\right)^{2}\right)\right) \\
& /\left(R_{C}^{6}\left(\left(\epsilon_{B}^{\prime}-\epsilon_{C}^{\prime}\right)^{2}+\left(\epsilon_{B}^{\prime \prime}-\epsilon_{C}^{\prime \prime}\right)^{2}\right)\right. \\
& +2 R_{B}^{3} R_{C}^{3}\left(\left(\epsilon_{B}^{\prime}-\epsilon_{C}^{\prime}\right)\left(2 \epsilon_{B}^{\prime}+\epsilon_{C}^{\prime}\right)+\left(\epsilon_{B}^{\prime \prime}-\epsilon_{C}^{\prime \prime}\right)\left(2 \epsilon_{B}^{\prime \prime}+\epsilon_{C}^{\prime \prime}\right)\right) \\
& \left.+R_{B}^{6}\left(\left(2 \epsilon_{B}^{\prime}+\epsilon_{C}^{\prime}\right)^{2}+\left(2 \epsilon_{B}^{\prime \prime}+\epsilon_{C}^{\prime \prime}\right)^{2}\right)\right) .
\end{aligned}
$$

In this simple case, the values for the constants in Eqs. (C11)(C15) can be substituted analytically into Eq. (C20) yielding an expression for $\epsilon_{\mathrm{eff}}^{\prime \prime}$ which is identical to Eq. (C23).

\section{APPENDIX D: DERIVATION OF EQ. (13) OF THE MAIN TEXT}

We start by calculating the position of the absorption maximum in the parallel case beginning from Eq. (2) and inserting an exponentially decaying correlation function $\Phi_{\|}(t)=A_{\|} e^{-t / \tau_{\|}}$. This leads to the well-known Debye form for the dielectric function

$$
\epsilon_{\|}=\frac{A_{\|}}{1+i 2 \pi f \tau_{\|}}+1,
$$

where $\epsilon_{\|}=\epsilon_{\|}^{\prime}-i \epsilon_{\|}^{\prime \prime}$ is a complex number containing real and imaginary parts of the dielectric function. The derivative of the imaginary part with respect to $f$ follows as

$$
\frac{d \epsilon_{\|}^{\prime \prime}}{d f}=\frac{A_{\|}\left(2 \pi \tau_{\|}-8 \pi^{3} f^{2} \tau_{\|}^{3}\right)}{\left(1+4 \pi^{2} f^{2} \tau_{\|}^{2}\right)^{2}},
$$

giving for the position of the absorption maximum in $\epsilon_{\|}^{\prime \prime}$,

$$
f_{\max , \|}=\frac{1}{2 \pi \tau_{\|}} .
$$

A similar calculation for the perpendicular component starts from Eq. (3) of the main text which after inserting $\Phi_{\perp}(t)=A_{\perp} e^{-t / \tau_{\perp}}$ gives

$$
\epsilon_{\perp}=\frac{1}{1-A_{\perp}\left(1-\frac{i 2 \pi f \tau_{\perp}}{1+i 2 \pi f \tau_{\perp}}\right)} .
$$

Taking again the derivative of the imaginary part with respect to $f$ gives

$$
\frac{d \epsilon_{\perp}^{\prime \prime}}{d f}=\frac{A_{\perp} 2 \pi \tau_{\perp}\left(\left(-1+A_{\perp}\right)^{2}-4 \pi^{2} f^{2} \tau_{\perp}^{2}\right)}{\left(\left(-1+A_{\perp}\right)^{2}+4 \pi^{2} f^{2} \tau_{\perp}^{2}\right)^{2}}
$$

and finally

$$
f_{\max , \perp}=\frac{1-A_{\perp}}{2 \pi \tau_{\perp}}=\frac{1}{\epsilon_{\perp}^{\prime}(f=0)} \frac{1}{2 \pi \tau_{\perp}},
$$

where the last identity can be inferred from Eq. (3) of the main text with $f=0$.
${ }^{1}$ B. Bagchi, "Water dynamics in the hydration layer around proteins and micelles," Chem. Rev. 105, 3197 (2005).

${ }^{2}$ R. Buchner and G. Hefter, "Interactions and dynamics in electrolyte solutions by dielectric spectroscopy," Phys. Chem. Chem. Phys. 11, 8984-8999 (2009)

${ }^{3}$ B. Born, H. Weingärtner, E. Bründermann, and M. Havenith, "Solvation dynamics of model peptides probed by terahertz spectroscopy: Observation of the onset of collective network motions," J. Am. Chem. Soc 131, 3752 (2009)

${ }^{4}$ K. J. Tielrooij, D. Paparo, L. Piatkowski, H. J. Bakker, and M. Bonn, "Dielectric relaxation dynamics of water in model membranes probed by terahertz spectroscopy,” Biophys. J. 97, 2484-2492 (2009).

${ }^{5} \mathrm{U}$. Kaatze, "Bound water: Evidence from and implications for the dielectric properties of aqueous solutions," J. Mol. Liq. 162, 105 (2011).

${ }^{6}$ T. Fukasawa, T. Sato, J. Watanabe, Y. Hama, W. Kunz, and R. Buchner, "Relation between dielectric and low-frequency Raman spectra for hydrogen-bond liquids," Phys. Rev. Lett. 95, 197802 (2005).

${ }^{7}$ I. R. Piletic, D. E. Moilanen, D. B. Spry, N. E. Levinger, and M. D. Fayer, "Testing the core/shell model of nanoconfined water in reverse micelles using linear and nonlinear IR spectroscopy," J. Phys. Chem. A 110, 49854999 (2006).

${ }^{8}$ J. Qvist and B. Halle, "Thermal signature of hydrophobic hydration dynamics," J. Am. Chem. Soc. 130, 10345-10353 (2008).

${ }^{9}$ A. A. Bakulin, M. S. Pshenichnikov, H. J. Bakker, and C. Petersen, "Hydrophobic molecules slow down the hydrogen-bond dynamics of water," J. Phys. Chem. A 115, 1821 (2011).

${ }^{10}$ R. Abseher, H. Schreiber, and O. Steinhauser, "The influence of a protein on water dynamics in its vicinity investigated by molecular dynamics simulation," Proteins 25, 366-378 (1996).

${ }^{11}$ R. Iftimie and M. E. Tuckerman, "Decomposing total IR spectra of aqueous systems into solute and solvent contributions: A computational approach using maximally localized Wannier orbitals," J. Chem. Phys. 122, 214508 (2005).

${ }^{12}$ M. Heyden, J. Sun, S. Funkner, G. Mathias, H. Forbert, M. Havenith, and D. Marx, "Dissecting the THz spectrum of liquid water from first principles via correlations in time and space," Proc. Natl. Acad. Sci. U.S.A. 107, 12068 (2010).

${ }^{13} \mathrm{M}$. Heyden and M. Havenith, "Combining $\mathrm{THz}$ spectroscopy and MD simulations to study protein-hydration coupling," Methods 52, 74 (2010).

${ }^{14}$ T. Yamaguchi, H. Furuhashi, T. Matsuoka, and S. Koda, "Dynamic solvophobic effects and its cooperativity in the hydrogen-bonding liquids studied by dielectric and nuclear magnetic resonance relaxation," J. Phys. Chem. B 112, 16633-16641 (2008).

${ }^{15}$ K. J. Tielrooij, J. Hunger, R. Buchner, M. Bonn, and H. J. Bakker, "Influence of concentration and temperature on the dynamics of water in the hydrophobic hydration shell of tetramethylurea," J. Am. Chem. Soc. 132, 15671-15678 (2010).

${ }^{16}$ R. K. Murarka and T. Head-Gordon, "Dielectric relaxation of aqueous solutions of hydrophilic versus amphiphilic peptides," J. Phys. Chem. B 112, 179 (2008).

${ }^{17}$ J. C. Rasaiah, S. Garde, and G. Hummer, "Water in nonpolar confinement: From nanotubes to proteins and beyond," Annu. Rev. Phys. Chem. 59, 713 (2008).

${ }^{18} \mathrm{~J}$. Li, J. M. Fernandez, and B. J. Berne, "Waters role in the forceinduced unfolding of ubiquitin," Proc. Natl. Acad. Sci. U.S.A. 107, 19284 (2010).

${ }^{19}$ R. Baron, P. Setny, and J. A. McCammon, "Water in cavity-ligand recognition," J. Am. Chem. Soc. 132, 12091 (2010).

${ }^{20}$ D. Thirumalai, G. Reddy, and J. E. Straub, "Role of water in protein aggregation and amyloid polymorphism," Acc. Chem. Res. 45, 83 (2012).

${ }^{21}$ D. Laage, G. Stirnemann, and J. T. Hynes, "Why water reorientation slows without iceberg formation around hydrophoic solutes," J. Phys. Chem. B 113, 2428 (2009).

${ }^{22} \mathrm{~A}$. Chandra and B. Bagchi, "Microscopic expression for frequency and wave vector dependent dielectric constant of a dipolar fluid," J. Chem. Phys. 90, 1832 (1988).

${ }^{23}$ F. O. Raineri, B.-C. Perng, and H. L. Friedman, "A fluctuating charge density formulation of the dielectric behavior of liquids - with applications to equilibrium and nonequilibrium solvation," Electrochim. Acta 42, 2749 (1997).

${ }^{24}$ P. A. Bopp, A. A. Kornyshev, and G. Suttman, "Freqency and wave-vector dependent dielectric function of water: Collective modes and relaxation spectra," J. Chem. Phys. 109, 1939 (1998). 
${ }^{25}$ S. Schrödle, G. Hefter, W. Kunz, and R. Buchner, "Effects of nonionic surfactant $\mathrm{C}_{12} \mathrm{E}_{5}$ on the cooperative dynamics of water," Langmuir 22, 924 (2006).

${ }^{26}$ B. Klösgen, C. Reichle, S. Kohlsmann, and K. D. Kramer, "Dielectric spectroscopy as a sensor of membrane headgroup mobility," Biophys. J. 71, 3251 (1996).

${ }^{27}$ P. B. Petersen and R. J. Saykally, "Probing the interfacial structure of aqueous electrolytes with femtosecond second harmonic generation spectroscopy," J. Phys. Chem. B 110, 14060 (2006).

${ }^{28}$ Y. Rao, D. Song, N. J. Turro, and K. B. Eisenthal, "Orientational motions of vibrational chromophores in molecules at the air/water interface with time-resolved sum frequency generation," J. Phys. Chem. B 112, 13572 (2008).

${ }^{29}$ M. Sajadi, Y. Ajaj, I. Ioffe, H. Weingärtner, and N. P. Ernsting, "Terahertz absorption spectroscopy of a liquid using a polarity probe: A case study of trehalose/water mixtures," Angew. Chem., Int. Ed. 49, 454 (2010).

${ }^{30}$ C. Sendner, D. Horinek, L. Bocquet, and R. R. Netz, "Interfacial water at hydrophobic and hydrophilic surfaces: Slip, viscosity and diffusion," Langmuir 25, 10768 (2009)

${ }^{31}$ F. Sedlmeier, Y. von Hansen, L. Mengyu, D. Horinek, and R. R. Netz, "Water dynamics at interfaces and solutes: Disentangling free energy and diffusivity contributions," J. Stat. Phys. 145, 240-252 (2011).

${ }^{32}$ H. J. C. Berendsen, J. R. Grigera, and T. P. Straatsma, "The missing term in effective pair potentials," J. Phys. Chem. 91, 6269 (1987).

${ }^{33}$ M. Neumann, "Dielectric relaxation in water: Computer simulations with the TIP4P potential," J. Chem. Phys. 85, 1567 (1986).

${ }^{34}$ D. van der Spoel, E. Lindahl, B. Hess, G. Groenhof, A. E. Mark, and H. J. C. Berendsen, "GROMACs: Fast, flexible, and free," J. Comput. Chem. 26, 1701 (2005)

${ }^{35}$ S. Plimpton, "Fast parallel algorithms for short-range molecular dynamics," J. Comput. Phys. 117, 1-17 (1995).

${ }^{36}$ H. J. C. Berendsen, J. P. M. Postma, W. F. van Gunsteren, A. DiNola, and J. R. Haak, "Molecular dynamics with coupling to an external bath," J. Chem. Phys. 81, 3684 (1984).

${ }^{37}$ I. Yeh and M. L. Berkowitz, "Ewald summation for systems with slab geometry,” J. Chem. Phys. 111, 3155-3162 (1999).

${ }^{38}$ C. J. F. Böttcher and P. Bordewijk, Theory of Electric Polarization II (Elsevier Scientific, 1978)
${ }^{39}$ H. A. Stern and S. E. Feller, "Calculation of the dielectric permittivity profile for a nonuniform system: Application to a lipid bilayer system," J. Chem. Phys. 118, 3401 (2003).

${ }^{40}$ D. J. Bonthuis, S. Gekle, and R. R. Netz, "Dielectric profile of interfacial water and its effect on double layer capacitance," Phys. Rev. Lett. 107, 166102 (2011).

${ }^{41}$ D. J. Bonthuis, S. Gekle, and R. R. Netz, "Profile of the static permittivity tensor of water at interfaces: Consequences for capacitance, hydration interaction and ion adsorption," Langmuir 28, 7679-7694 (2012).

${ }^{42}$ V. A. Froltsov and S. H. L. Klapp, "Dielectric response of polar fluids in narrow slit pores," J. Chem. Phys. 126, 114703 (2007).

${ }^{43}$ See supplementary material at http://dx.doi.org/10.1063/1.4749380 for additional data.

${ }^{44}$ M. R. Reddy and M. Berkowitz, "The dielectric constant of SPC/E water," Chem. Phys. Lett. 155, 173 (1989).

${ }^{45}$ L. K. H. van Beek, Prog. Dielectr. 7, 69 (1965).

${ }^{46}$ T. Hanai and K. Sekine, "Theory of dielectric relaxations due to the interfacial polarization for two-component suspensions of spheres," Colloid Polym. Sci. 264, 888 (1986).

${ }^{47}$ J. E. W. Castner, G. R. Fleming, B. Bagchi, and M. Maroncelli, "The dynamics of polar solvation: Inhomogenepous dielectric continuum models," J. Chem. Phys. 89, 3519 (1988).

${ }^{48}$ The core radius is obtained from $4 / 3 \pi R_{c}^{3}=V_{p}$ with the particle volume given by $V_{p}=M_{p} / \rho$ where the density $\rho=0.968 \mathrm{~g} / \mathrm{cm}^{3}$ for pure TMU and the particle mass in turn is calculated from $M_{p}=M / N_{A}$ with the molar mass of TMU $M=116.16 \mathrm{~g} / \mathrm{mol}$ and $N_{A}$ Avogadro's constant.

${ }^{49}$ O. Glatter, G. Fritz, H. Lindner, J. Brunner-Popela, R. Mittelbach, R. Strey, and S. U. Egelhaaf, "Nonionic micelles near the critical point: micellar growth and attractive interaction," Langmuir 16, 8692 (2000).

${ }^{50}$ M. Maroncelli and G. R. Fleming, "Computer simulation of the dynamics of aqueous solvation,” J. Chem. Phys. 89, 5044 (1988).

${ }^{51}$ G. Stirnemann, S. R.-V. Castrillón, J. T. Hynes, P. J. Rossky, P. G. Debenedetti, and D. Laage, "Non-monotonic dependence of water reorientation dynamics on surface hydrophilicity: Competing effects of the hydration structure and hydrogen-bond strength," Phys. Chem. Chem. Phys. 13, 19911 (2011).

${ }^{52}$ J. J. Loparo, C. J. Fecko, J. D. Eaves, S. T. Roberts, and A. Tokmakoff, "Reorientational and configurational fluctuations in water observed on molecular length scales," Phys. Rev. B 70, 180201 (2004). 